the determinant $\left|A_{k l}\right|$ must vanish. This is the announced identity, connecting two different (not linearly dependent) Heine-Stieltjes polynomials $P_{n}^{\left(\alpha_{0}, \cdots, \alpha_{p}\right)}(x)$ and $P_{n^{\prime}}^{\left(\alpha_{0}, \cdots, \alpha_{p}\right)}(x), n \neq n^{\prime}$, or $n=n^{\prime}$, but $\left(n_{1}, n_{2}, \cdots, n_{p}\right) \neq\left(n_{1}^{\prime}, n_{2}^{\prime}, \cdots, n_{p}^{\prime}\right)$. For $p=1$ (2) goes over in the known orthogonality relations

$$
\int_{-1}^{+1} P_{n}^{\left(\alpha_{0}, \alpha_{1}\right)}(x) \cdot P_{n^{\prime}}^{\left(\alpha_{0}, \alpha_{1}\right)}(x)\left|(x+1)^{\alpha_{0}}(x-1)^{\alpha_{1}}\right|=0
$$

with $\left|(x+1)^{\alpha_{0}}(x-1)^{\alpha_{1}}\right|$ as weight function. We call $\mid\left(x-a_{0}\right)^{\alpha_{0}} \ldots$ $\left(x-a_{p}\right)^{\alpha_{p}} \mid$ still the weight function of the generalized Jacobi polynomials of the order $p$.

By introduction of the extended Heine-Stieltjes polynomials $p_{m}^{\left(\alpha_{0}, \cdots, \alpha_{p}\right)}(x)=\left(x-a_{0}\right)^{\alpha_{0}} \cdots\left(x-a_{p}\right)^{\alpha_{p}} P_{n}^{\left(\alpha_{0}, \cdots \alpha_{p}\right)}(x)$ and by denoting $\int_{\alpha_{k}}^{\alpha_{k}+1}\left(u v x^{l} /\left(x-a_{0}\right)^{\alpha_{0}} \cdots\left(x-a_{p}\right)^{\alpha_{p}}\right) d x$, by $B_{k l}$, we obtain $\left|B_{k l}\right|=0 .{ }^{12}$

We turn finally to the special case, interesting among others for the theory of the transfinite diameter and the Fekete-diameters of a finite degree $m$, that $\alpha_{i}=\rho_{i}=1, i=0, \cdots, p$. Then the generalized Vandermondean $V_{m}\left(\xi_{1}, \xi_{2}, \cdots, \xi_{m} ; e_{1}, e_{2}, \cdots, e_{m}\right)$ becomes the usual Vandermondean $V_{m}\left(\xi_{1}, \xi_{2}, \cdots, \xi_{m}\right)=\prod_{1 i_{i<k} m}\left(\xi_{i}-\xi_{k}\right)$ and, since $A_{1}(x)=A(x)$, there results $B(x)=A^{\prime}(x)$.

Mathematical Institute of the Technical University of Berlin

$12 u$ and $v$ are two different extended Heine-Stieltjes polynomials with the same superscripts $\alpha_{0}, \alpha_{1}, \cdots, \alpha_{p}$. The weight-function corresponding to them is $\left|\left(x-a_{0}\right)^{-\alpha_{0}}\left(x-a_{1}\right)^{-\alpha_{1}} \cdots\left(x-a_{p}\right)^{-\alpha_{p}}\right|=\left|\left(x-a_{0}\right)^{\alpha_{0}} \cdots\left(x-a_{p}\right)^{\alpha_{p}}\right|^{-1}$.

\title{
AN INTEGRAL TRANSFORMATION RELATION
}

\section{R. G. BUSCHMAN}

In a recent paper Rooney [3] has discussed the interesting formula

$$
\int_{0}^{\infty} t^{\nu+1} K_{\nu}(s t) \phi\left(t^{2}\right) d t=2^{\nu-1} s^{-\nu} \int_{0}^{\infty} e^{-y} y^{\nu-1} f\left(s^{2} / 4 y\right) d y
$$

as a generalization of the formula for the Laplace transformation of $t^{\nu} \phi\left(t^{2}\right)$. Here $K_{\nu}(x)$ is the modified Bessel function of the second kind and

$$
f(s)=\int_{0}^{\infty} e^{-s t} \phi(t) d t=\mathscr{L}\{\phi(t)\}
$$

Received by the editors March 12, 1958 and, in revised form, May 7, 1958. 
In this paper we generalize further the relations for the integral transform of $k(t) F[g(t)]$ obtained in [1] and then show that the above formula can be obtained by choosing specific functions.

Suppose that for some kernel $K(s, t)$

$$
\int_{0}^{\infty} K(s, t) k(t) F[g(t)] d t=\mathfrak{K}\{k(t) F[g(t)]\}
$$

exists. If $g$ and its inverse function $h=g^{-1}$ are monotonic, differentiable, and real on $(0, \infty)$ and $g(0)=0, g(\infty)=\infty$ (or $g(0)=\infty, g(\infty)$ $=0)$, then

$$
\mathfrak{K}\{k(t) F[g(t)]\}=\int_{0}^{\infty} K[s, h(p)] k[h(p)]\left|h^{\prime}(p)\right| F(p) d p .
$$

Next we consider a symmetric kernel $S(u, p) \equiv S(p, u)$. If there is a function $\Phi(s, u)$ such that

$$
\phi(s, p)=\int_{0}^{\infty} S(u, p) \Phi(s, u) d u=s\{\Phi(s, u)\}
$$

where

$$
\phi(s, p)=K[s, h(p)] k[h(p)]\left|h^{\prime}(p)\right|,
$$

then we obtain

$$
\mathcal{K}\{k(t) F[g(t)]\}=\int_{0}^{\infty}\left(\int_{0}^{\infty} S(u, p) \Phi(s, u) d u\right) F(p) d p .
$$

Now, provided that the function $F(p)$ is such that the interchange of integration is valid and that $s\{F(p)\}=f(u)$, then

$$
\mathfrak{K}\{k(t) F[g(t)]\}=\int_{0}^{\infty} \Phi(s, u) f(u) d u .
$$

As an example, the formula of Rooney can now be obtained by letting $K(s, t)=K_{\nu}(s t), S(u, p)=e^{-u p}, F(t)=\phi(t), g(t)=t^{2}, k(t)=t^{\nu+1}$, and $\Phi(s, u)$ can then be obtained from tables $[2,5.16(40)]$.

The known relation for the Laplace transformation

$$
\mathfrak{L}\left\{t^{\nu-1} F(1 / t)\right\}=\int_{0}^{\infty}(u / s)^{\nu / 2} J_{\nu}\left[2(s u)^{1 / 2}\right] f(u) d u,
$$

where $\mathscr{L}\{F(t)\}=f(s)$, is obtained by choosing $K(s, t)=e^{-s t}, S(u, p)$ $=e^{-u p}, g(t)=t^{-1}$, and $k(t)=t^{\nu-1} . \Phi(s, u)$ is then obtained from [2, $5.6(40)]$. 
For another example consider the parabolic cylinder function and let $K(s, t)=t^{2 v} e^{s^{2} t^{2} / 4} D_{-2 v}(s t), S(u, p)=e^{-u p}, g(t)=t^{-2}$, and $k(t)=t^{-3}$. $\Phi(s, u)$ is then obtained from $[2,5.18(7)]$ so that after some simplification we have

$$
\int_{0}^{\infty} t^{2 \nu-3} e^{s^{2} t^{2} / 4} D_{-2 \nu}(s t) F\left(t^{-2}\right) d t=[2 \Gamma(2 \nu)]^{-1} \int_{0}^{\infty} u^{2 \nu-1} e^{-s u} f\left(u^{2} / 2\right) d u,
$$

in which $\mathscr{L}\{F(t)\}=f(u)$. In particular if $F(t)=t^{-3 / 2} e^{-b / 4 t}$, then $f(u)$ $=2 \pi^{1 / 2} b^{-1 / 2} e^{-b^{1 / 2} u^{1 / 2}}$ and

$$
\begin{aligned}
\int_{0}^{\infty} e^{-b t^{2} / 4} t^{2 \nu} e^{s^{2} t^{2} / 4} D_{-2 \nu}(s t) d t & \\
& =\pi^{1 / 2}\left[b^{1 / 2} \Gamma(2 \nu)\right]^{-1} \int_{0}^{\infty} u^{2 \nu-1} \exp \left\{-\left[s+(b / 2)^{1 / 2}\right] u\right\} d u \\
& =\pi^{1 / 2} b^{-1 / 2}\left[s+(b / 2)^{1 / 2}\right]^{-2}
\end{aligned}
$$

for $\nu>0, b>0, s>0$.

To summarize, under the conditions stated above, if

$$
s\{\Phi(s, u)\}=\phi(s, p) \text { and } s\{F(u)\}=f(p)
$$

where

$$
\phi(s, p)=K[s, h(p)] k[h(p)]\left|h^{\prime}(p)\right|,
$$

then

$$
\Re\{k(t) F[g(t)]\}=\int_{0}^{\infty} \Phi(s, u) f(u) d u .
$$

\section{REFERENCES}

1. R. G. Buschman, A substitution theorem for the Laplace transformation and its generalization to transformations with symmetric kernel, Pacific J. Math. vol. 7 (1957) pp. 1529-1533.

2. A. Erdélyi et al., Tables of integral transforms, New York, 1954.

3. P. G. Rooney, A property of the Laplace transformation, Proc. Amer. Math. Soc. vol. 8 (1957) pp. 883-886.

UNIVERSITY OF Wichita 